\title{
Counting pop-stacked permutations in polynomial time
}

\author{
Anders Claesson \\ Division of Mathematics \\ The Science Institute \\ University of Iceland \\ akc@hi.is
}

\author{
Bjarki Ágúst Guðmundsson \\ Department of Computer Science \\ Reykjavík University \\ Reykjavík, Iceland \\ bjarkig@ru.is
}

\author{
Jay Pantone \\ Department of Mathematical and Statistical Sciences \\ Marquette University \\ Milwaukee, WI, USA \\ jay.pantone@marquette.edu
}

\begin{abstract}
Permutations in the image of the pop-stack operator are said to be pop-stacked. We give a polynomial-time algorithm to count pop-stacked permutations up to a fixed length and we use it to compute the first 1000 terms of the corresponding counting sequence. Only the first 16 terms had previously been computed. With the 1000 terms we prove some negative results concerning the nature of the generating function for pop-stacked permutations. We also predict the asymptotic behavior of the counting sequence using differential approximation.
\end{abstract}

\section{Pop-stacked permutations}

The abstract data type known as a stack has two operations: push adds an element at the top of the stack; pop removes the top element from the stack. A pop-stack is a variation of this introduced by Avis and Newborn [2] in which the pop operation empties the entire stack.

Let $\pi=a_{1} a_{2} \ldots a_{n}$ be a permutation of $[n]=\{1,2, \ldots, n\}$. An ascending run of $\pi$ is a maximal sequence of consecutive ascending letters $a_{i}<a_{i+1}<\cdots<$ $a_{i+d-1}$, and a descending run is defined similarly. For instance, the ascending runs of $\pi=617849235$ are $6,178,49$ and 235; its descending runs are $61,7,84$, 92,3 and 5 .

Let $P(\pi)$ be the result of greedily sorting $\pi$ using a pop-stack subject to the constraint that elements on the pop-stack are increasing when read from the 
top to the bottom of the stack. In other words, if we factor $\pi$ into its descending runs $\pi=D_{1} D_{2} \ldots D_{m}$, then $P(\pi)$ is obtained by reversing each of those runs: $P(\pi)=D_{1}^{r} D_{2}^{r} \ldots D_{m}^{r}$. For instance, $P(5321764)=1235467$ and $P(617849235)=167482935$. A permutation $\pi$ is said to be sortable by a popstack if $P(\pi)$ is the identity permutation. More generally, $\pi$ is said to be sortable by $k$ passes through a pop-stack if $P^{k}(\pi)$ is the identity permutation. Claesson and Guðmundsson 3] showed that the generating function for the number of permutations of $[n]$ that are sortable by $k$ passes through a pop-stack is always rational.

Asinowski et al. [1] defined that $\sigma$ is pop-stacked if $\sigma=P(\pi)$ for some permutation $\pi$, and gave the following theorem.

Theorem 1 (Asinowski et al. [1]). A permutation is pop-stacked if and only if for each pair $\left(R_{i}, R_{i+1}\right)$ of its adjacent ascending runs $\min R_{i}<\max R_{i+1}$.

They further showed that the generating function for pop-stacked permutations of $[n]$ with exactly $k$ ascending runs is rational for each $k$. Enumerating popstacked permutations without this restriction is, however, an open problem. Asinowski et al. initiated an investigation into this by calculating the number of pop-stacked permutations of length $n=1, \ldots, 16$, adding the resulting sequence to the OEIS [11] as A307030 and noting that "this sequence is hard to compute". In the following section, we give an efficient algorithm for counting pop-stacked permutations, expanding the sequence up to $n=1000$. While the algorithm and the augmented sequence could give additional insight into the structure of popstacked permutations, finding a generating function or a closed form solution to their enumeration remains an open problem. Section 3 gives experimental data in this direction.

\section{Polynomial-time counting algorithm}

A ballot, alternatively known as an ordered set partition, is a collection of pairwise disjoint nonempty sets, referred to as blocks, where the blocks are assigned some total ordering. Any permutation can be seen as a ballot by decomposing it into its ascending runs. The permutation $\pi=617849235$ would then be viewed as the ballot $\{6\}\{1,7,8\}\{4,9\}\{2,3,5\}$. Conversely, a ballot $B_{1} B_{2} \ldots B_{k}$ represents a permutation in this manner if, and only if, $\max B_{i}>\min B_{i+1}$ for each $i$ in $[k-1]$. Thus, the ballots corresponding to pop-stacked permutations are precisely those such that

$$
\max B_{i}>\min B_{i+1} \quad \text { and } \quad \min B_{i}<\max B_{i+1} .
$$

In other words, the intervals between the smallest and largest elements of each pair of adjacent blocks overlap,

$$
\left[\min B_{i}, \max B_{i}\right] \cap\left[\min B_{i+1}, \max B_{i+1}\right] \neq \emptyset,
$$

and we call these ballots overlapping; here, $[a, b]$ denotes the interval $\{a, a+$ $1, \ldots, b\}$. Let $F[U]$ be the set of overlapping ballots whose underlying set is $U$. 
As an example,

$$
F[\{1,2,3\}]=\{\{1,2,3\},\{2\}\{1,3\},\{1,3\}\{2\}\} .
$$

Let $F_{c, d}[U]$ denote the subset of $F[U]$ whose last block, $B$, is such that $c=\min B$ and $d=\max B$. Clearly, if $c>d$ then $F_{c, d}[U]=\emptyset$. Also,

$$
F[U]=\bigcup_{c, d \in U} F_{c, d}[U] .
$$

If $c=\min U$ and $d=\max U$, then one possibility is that there is a single block consisting of all elements of $U$. Let us now consider the more typical case when there are two or more blocks, and let us write the ballot as $B_{1} B_{2} \ldots B_{k}$. By definition, its last block, $B_{k}$, satisfies $c=\min B_{k}$ and $d=\max B_{k}$, or expressed differently $\{c, d\} \subseteq B_{k} \subseteq[c, d]$. Let $a=\min B_{k-1}$ and $b=\max B_{k-1}$. The blocks $B_{k-1}$ and $B_{k}$ overlap if, and only if, $a<d$ and $b>c$. Thus

$$
\begin{gathered}
F_{c, d}[U]=\{U: c=\min U \wedge d=\max U\} \cup \\
\bigcup_{\substack{\{c, d\} \subseteq B \subseteq[c, d] \\
a, b \in U \backslash B \\
a<d \wedge b>c}} F_{a, b}[U \backslash B] B,
\end{gathered}
$$

where $F_{a, b}[U \backslash B] B$ is the set $\left\{w B: w \in F_{a, b}[U \backslash B]\right\}$, and the somewhat cryptic looking $\{U: c=\min U \wedge d=\max U\}$ expresses the singleton $\{U\}$ if $c=\min U$ and $d=\max U$, and the empty set otherwise.

We now turn to counting. Let $f(n)$ be the number of overlapping ballots of $[n]$. That is, $f(n)=|F[n]|$ in which $F[n]$ is short for $F[\{1, \ldots, n\}]$. Also, let $f_{c, d}(n)=\left|F_{c, d}[n]\right|$. If $c>d$ then $f_{c, d}(n)=0$. Otherwise we shall use the recursive decomposition (11) and do case analysis based on whether $c$ and $d$ are the same or two distinct elements.

If $c=d$, then the last block consists of a single point. In terms of (1) the ballot is written $w\{c\}$, where $w \in F_{a, b}[[n] \backslash\{c\}]$ and $a<c<b$. After "rescaling" we can consider $w$ a ballot in $F[n-1]$; here we subtract 1 from each element larger than $c$. Note that this, however, also lowers the value of $b$ by one. Thus, the number of such ballots is

$$
\sum_{a=1}^{c-1} \sum_{b=c}^{n-1} f_{a, b}(n-1)
$$

If $c<d$, then write the ballot as $w B$ and let $\ell=|B|-2$. There are $\left(\begin{array}{c}d-c-1 \\ \ell\end{array}\right)$ ways to choose $B$. After rescaling we have $w \in F_{a, b}[n-\ell-2]$, where $a \leq d-\ell-2$ and $b \geq c$. Thus, the number of such ballots is

$$
\sum_{\ell=0}^{d-c-1}\left(\begin{array}{c}
d-c-1 \\
\ell
\end{array}\right) \sum_{a=1}^{d-\ell-2} \sum_{b=c}^{n-\ell-2} f_{a, b}(n-\ell-2) .
$$

Finally, if $c=1$ and $d=n$, we also count the case where the ballot consists of 
a single block. Taking all this together, we have that

$$
\begin{aligned}
f_{c, d}(n) & =[c=1 \wedge d=n] \\
& +[c=d] \sum_{a=1}^{c-1} \sum_{b=c}^{n} f_{a, b}(n-1) \\
& +[c<d] \sum_{\ell=0}^{d-c-1}\left(\begin{array}{c}
d-c-1 \\
\ell
\end{array}\right) \sum_{a=1}^{d-\ell-2} \sum_{b=c}^{n-\ell-2} f_{a, b}(n-\ell-2) .
\end{aligned}
$$

Here $[p]$ is the Iverson bracket: it converts the proposition $p$ into 1 if $p$ is satisfied, and 0 otherwise. Further, $f(n)=\sum_{a=1}^{n} \sum_{b=a}^{n} f_{a, b}(n)$.

Recurrence (2) can be augmented to count overlapping ballots with a specific number of blocks, or, equivalently, pop-stacked permutations with a specific number of ascending runs. Let $f_{c, d}(n, k)$ denote the number of overlapping ballots of $[n]$ with exactly $k$ blocks. Then we have $f(n, k)=\sum_{a=1}^{n} \sum_{b=a}^{n} f_{a, b}(n, k)$ and

$$
\begin{aligned}
f_{c, d}(n, k) & =[c=1 \wedge d=n \wedge k=1] \\
& +[c=d] \sum_{a=1}^{c-1} \sum_{b=c}^{n} f_{a, b}(n-1, k-1) \\
& +[c<d] \sum_{\ell=0}^{d-c-1}\left(\begin{array}{c}
d-c-1 \\
\ell
\end{array}\right) \sum_{a=1}^{d-\ell-2} \sum_{b=c}^{n-\ell-2} f_{a, b}(n-\ell-2, k-1) .
\end{aligned}
$$

Note that there are two locations in the recurrence (2) where we have a plain two-dimensional sum over $f$, that is $\sum_{a=\star}^{\star} \sum_{b=\star}^{\star} f_{a, b}(\star)$, where $\star$ are fixed and not dependent on $a, b$ or each other. We simplify these two-dimensional sums using "prefix sums". Let

$$
g_{c, d}(n)=\sum_{a=1}^{c} \sum_{b=1}^{d} f_{a, b}(n)
$$

In particular, $g_{c, d}(n)=0$ if $c=0$ or $d=0$. Note that

$$
g_{c, d}(n)=f_{c, d}(n)+g_{c-1, d}(n)+g_{c, d-1}(n)-g_{c-1, d-1}(n) .
$$

Also noting that

$$
\sum_{a=p}^{q} \sum_{b=r}^{s} f_{a, b}(n)=g_{q, s}(n)-g_{p-1, s}(n)-g_{q, r-1}(n)+g_{p-1, r-1}(n),
$$

we can now simplify the above equation to

$$
\begin{aligned}
f_{c, d}(n) & =[c=1 \wedge d=n] \\
& +[c=d] \Delta_{c-1, n, c-1}(n-1) \\
& +[c<d] \sum_{\ell=0}^{d-c-1}\left(\begin{array}{c}
d-c-1 \\
\ell
\end{array}\right) \Delta_{d-2-\ell, n-2-\ell, c-1}(n-2-\ell)
\end{aligned}
$$


where $\Delta_{u, v, w}(n)=g_{u, v}(n)-g_{u, w}(n)$. We further have $f(n)=g_{n, n}(n)$. The same simplification can also be applied to the recurrence for counting by blocks.

Say we wanted to compute $f(n)$ for all $1 \leq n \leq N$. We can precompute binomial coefficients $\left(\begin{array}{l}n \\ k\end{array}\right)$ for all $0 \leq k \leq n \leq N$ using the recurrence $\left(\begin{array}{l}n \\ k\end{array}\right)=\left(\begin{array}{c}n-1 \\ k-1\end{array}\right)+\left(\begin{array}{c}n-1 \\ k\end{array}\right)$. Then, using dynamic programming we can compute $f_{c, d}(n), g_{c, d}(n)$ and $f(n)$ using Recurrences 4 and 5 for all $1 \leq c, d \leq n \leq N$ in $O\left(N^{4}\right)$ time using $O\left(N^{3}\right)$ memory. When counting by blocks this is $O\left(N^{5}\right)$ time, but $O\left(N^{3}\right)$ memory is still sufficient.

This assumes that all arithmetic operations are $O(1)$. In reality, some of the numbers are on the order of $N$ !. This means that multiprecision arithmetic has to be used, which slows down the computation considerably. One way to speed this up is to choose a set of relatively small primes whose product is greater than $N$ !. For each prime $p$, the above computation is then carried out in the finite field $\mathbf{F}_{p}$. This can be done in parallel, as the computation for different primes is independent. The values of $f(n)$, which are guaranteed to be at most $N$ ! for all $n \leq N$, are then recovered using the Chinese Remainder Theorem.

This was used to calculate the number of pop-stacked permutations of each length up to $N=1000$. With 286 distinct primes just under $10^{9}$, and one CPU core per prime, the computation took just under an hour to complete, with each core using 3.8GiB of RAM. In a similar manner the number of pop-stacked permutations of each length up to $N=300$ grouped by number of ascending runs were computed. Table 1 gives the number of pop-stacked permutations of each length up to $N=45$, but the complete results, along with the code used to generate the results, can be found on GitHub [4].

\section{$3 \quad$ Experimental analysis}

With the first 1000 terms of the counting sequence of pop-stacked permutations now calculated, we turn to a pair of experimental techniques for an empirical analysis: automated fitting and differential approximation. Given initial terms of a counting sequence, the first of these methods searches for a generating function whose power series expansion matches the sequence, while the second predicts the asymptotic growth of the sequence.

For the counting sequence at hand, automated fitting does not conjecture a generating function, giving instead several (rigorous) negative results, while differential approximation gives very precise estimates of the asymptotic behavior.

\subsection{Automated fitting for pop-stacked permutations}

Let $a_{0}, a_{1}, \ldots$ be a counting sequence and $F(x)=\sum_{n \geq 0} a_{n} x^{n}$ its generating function. If $F(x)$ is a rational function, then we can write $F(x)=p(x) / q(x)$ for relatively prime polynomials $p(x), q(x) \in \mathbb{Q}[x]$; equivalently,

$$
q(x) F(x)-p(x)=0 .
$$




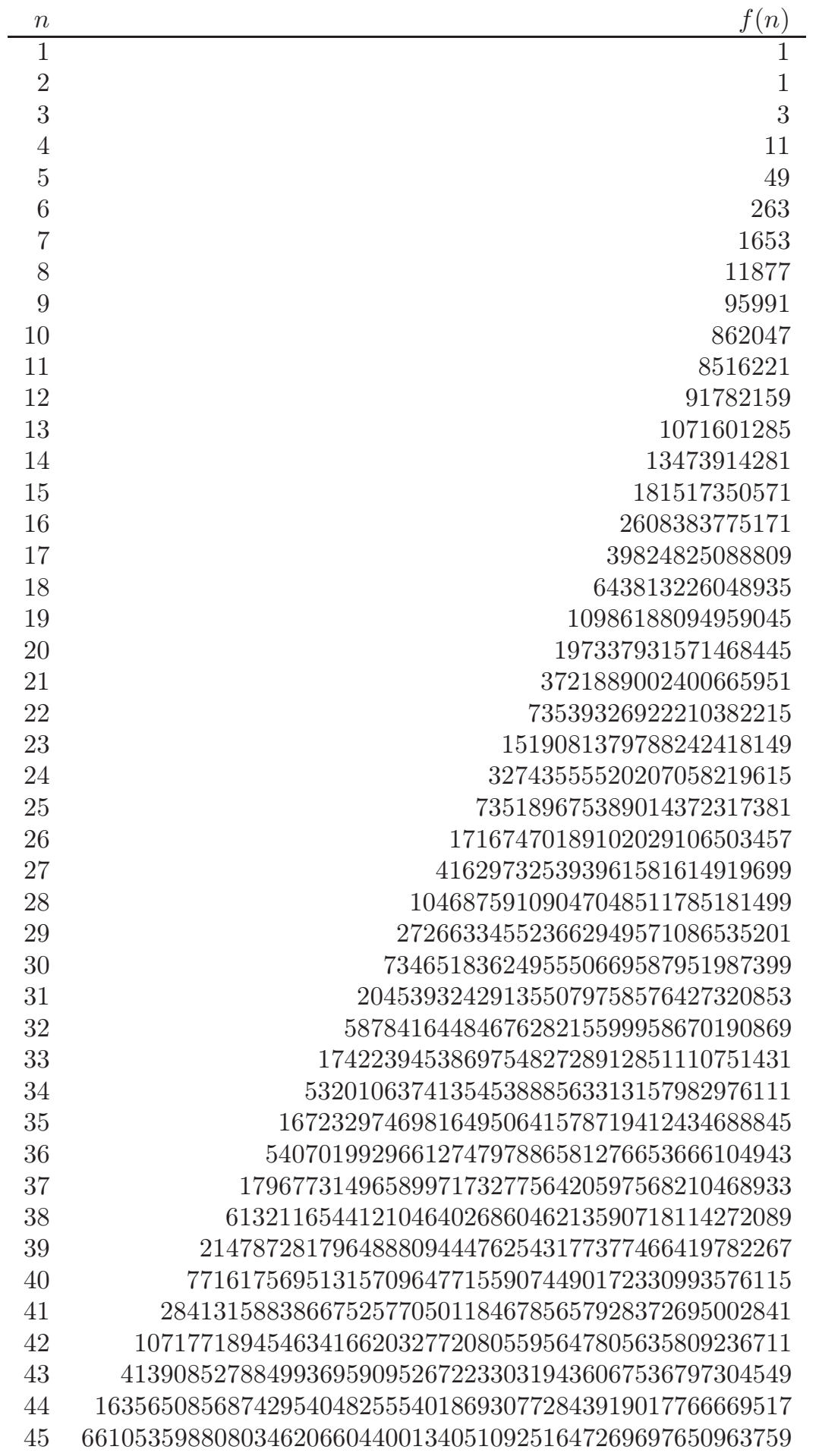

Table 1: The number of pop-stacked permutations of each length up to $N=45$. 
Conversely, suppose we are given only some initial terms $a_{0}, a_{1}, \ldots, a_{n}$ of a counting sequence and want to determine whether the generating function $F(x)$ of the unknown counting sequence is rational. If $F(x)$ is rational with $\max (\operatorname{deg}(p(x)), \operatorname{deg}(q(x)))=d$, then we can write Equation (6) as

$$
\left(q_{0}+q_{1} x+\cdots+q_{d} x^{d}\right)\left(a_{0}+a_{1} x+\cdots+a_{n} x^{n}\right)-\left(p_{0}+p_{1} x+\cdots+p_{d} x^{d}\right)=0 .
$$

Expanding the left-hand side gives a polynomial in $x$, and the coefficients of $x^{0}, x^{1}, \ldots, x^{n}$ must all equal 0 . We thus have a system of $n+1$ equations in the $2 d+2$ unknowns $p_{0}, \ldots, p_{d}, q_{0}, \ldots, q_{d}$. A generic system of this form is likely to have non-trivial solutions when $n \leq 2 d$, and so when initial terms up to $a_{n}$ are known, it is only productive to consider $d$ such that $2 d<n$.

If this system has no non-trivial solution, then we are guaranteed that $F(x)$ is not rational with numerator and denominator of degree at most $d$. If the system does have a non-trivial solution, then it is possible, though far from guaranteed, that

$$
F(x)=\frac{p_{0}+p_{1} x+\cdots+p_{d} x^{d}}{q_{0}+q_{1} x+\cdots+q_{d} x^{d}} .
$$

The larger the difference between $n$ and $2 d$, the more confident that one can be in such a conjecture. Empirically, this is like using the first $2 d$ known terms to guess the rational generating function and the remaining $n-2 d$ as confirmation.

Automated fitting can be extended beyond the realm of rational generating functions. A generating function $F(x)$ is called algebraic if there are polynomials $p_{0}(x), \ldots, p_{m}(x) \in \mathbb{Q}[x]$ such that

$$
p_{m}(x) F^{m}(x)+\cdots+p_{1}(x) F(x)+p_{0}(x)=0,
$$

called differentially finite (or D-finite) if there are polynomials $p_{0}(x), \ldots, p_{k}(x), q(x) \in \mathbb{Q}[x]$ such that

$$
p_{k}(x) F^{(k)}(x)+\cdots+p_{1}(x) F^{\prime}(x)+p_{0}(x) F(x)+q(x)=0,
$$

and called differentially algebraic (or D-algebraic) if there exists a $(k+2)$-variate polynomial $P$ with coefficients in $\mathbb{Q}$ such that

$$
P\left(x, F(x), F^{\prime}(x), \ldots, F^{(k)}(x)\right)=0 .
$$

To determine whether a generating function $F(x)$ is algebraic given some initial terms, an equation similar to (7) can be set up assuming each $p_{i}(x)$ has degree at most $d$, giving a linear system with $n$ equations and $(m+1)(d+1)$ unknowns. In the D-finite case, the system has $(k+2)(d+1)$ unknowns. The D-algebraic case requires further assumptions about form - the ideas are similar, but not worth elaborating upon here. There are various software packages that perform fitting of this kind, including Gfun [10] in Maple, Guess 99 in Mathematica, and Guess [7] in FriCAS. We have used a different package, GuessFunc, written by the third author.

We applied automated fitting to the counting sequence of pop-stacked permutations up to length 1000, and found no conjectured rational, algebraic, D-finite, or D-algebraic form for the unknown generating function $F(x)$. From this we can conclude rigorously that, for example, 
$\diamond$ If $F(x)$ is rational, then either the degree of the denominator or the degree of the numerator is at least 500 .

$\diamond$ If $F(x)$ is algebraic, then the degree of algebraicity $m$ and the maximum degree of polynomial coefficient $d=\max \left(p_{0}(x), \ldots, p_{m}(x)\right)$ must satisfy $(m+1)(d+1)>1000$.

$\diamond$ If $F(x)$ is D-finite, then the differential order $k$ and the maximum degree of polynomial coefficient $d=\max \left(q(x), p_{0}(x), \ldots, p_{k}(x)\right)$ must satisfy $(k+$ $2)(d+1)>1000$

A similar negative result could be written for the D-algebraic case, although it would require further explanation of the structure of the corresponding search space.

One can also apply various transformations to the generating function before initiating the automated fitting procedure. In addition to trying to find a fit for the ordinary generating function $F(x)=\sum_{n>0} a_{n} x^{n}$, we also attempted to find a fit for the exponential generating function $\sum_{n \geq 0}\left(a_{n} / n !\right) x^{n}$, the reciprocal $1 / F(x)$, the compositional inverse $F(x)^{\langle-1\rangle}$, and also several combinations of these transformations. No results were found.

\subsection{Automated fitting for pop-stacked permutations with a fixed number of ascending runs}

Let $F_{k}(x)$ denote the power series for those pop-stacked permutations with precisely $k$ ascending runs. Asinowski et al. [1] showed that these permutations are in bijection with words from a regular language that is recognized by a certain deterministic finite automaton (DFA) $\mathcal{A}_{k}$, proving that $F_{k}(x)$ is rational. Furthermore, a system of linear equations can be derived from this DFA, whose solution gives $F_{k}(x)$. Deriving $F_{k}(x)$ in this way is only practical for small values of $k$, however, as the number of states in $\mathcal{A}_{k}$ grows exponentially with $k$.

As mentioned earlier, Recurrence (3) permits the fast computation of the counting sequence for pop-stacked permutations with a fixed number of ascending runs. This, along with the techniques of automated fitting gives rise to a different approach for finding $F_{k}(x)$, albeit heuristically 1 .

Using the counting sequence for pop-stacked permutations of length at most 300 with a fixed number of ascending runs, we were able to find a rational fit for each $F_{k}(x)$ for $k \leq 24$. We were further able to verify that the rational fits were exact for $k \leq 6$ by using the previously mentioned method based on Asinowski

\footnotetext{
${ }^{1}$ Given enough terms of the sequence, automated fitting will find $F_{k}(x)$. The number of terms required is the sum of the degrees of the numerator and denominator of $F_{k}(x)$, which is not known. An upper bound is twice the number of states in $\mathcal{A}_{k}$, which is exponential.
} 
et al. [1]. The first four generating functions follow.

$$
\begin{aligned}
& F_{1}(x)=\frac{x}{1-x}, \\
& F_{2}(x)=\frac{2 x^{3}}{(1-2 x)(1-x)^{2}}, \\
& F_{3}(x)=\frac{2 x^{4}\left(1+3 x-6 x^{2}\right)}{(1-3 x)(1-2 x)^{2}(1-3 x)^{3}}, \\
& F_{4}(x)=\frac{2 x^{6}\left(21-74 x+5 x^{2}+180 x^{3}-144 x^{4}\right)}{(1-4 x)(1-3 x)^{2}(1-2 x)^{3}(1-x)^{4}} .
\end{aligned}
$$

Based on this data, which can be found in full on GitHub [4, we pose the following conjecture.

Conjecture 2. For all $k$, the rational generating function $F_{k}(x)$ can be written as

$$
F_{k}(x)=N_{k}(x) / \prod_{i=1}^{k}(1-i x)^{k-i+1},
$$

where $N_{k}(x)$ is a polynomial of degree $k(k+1) / 2$, the same degree as the conjectured denominator.

\subsection{Differential approximation}

Differential approximation empirically estimates the asymptotic growth of a counting sequence based on its initial terms by using linear differential equations to model the unknown generating function and studying the complex singularities of solutions of those linear differential equations. Here we will only present the results of this analysis - for information about how differential approximation works we refer the reader to [5, 6].

The cornerstone of analytic combinatorics is the observation that the asymptotic behavior of a counting sequence is intimately connected to the singularities of its generating function when treated as a complex function. For example, the location of the singularities closest to the origin (the dominant singularities) roughly determine the exponential growth of the counting sequence, and the nature of those singularities determines the sub-exponential behavior.

The output of differential approximation is an estimate of the location and nature (specifically, the critical exponent) of all singularities of the unknown generating function based on the given known initial terms. Typically, although not always, the dominant singularity is predicted with the highest precision, with the precision of the estimates of other singularities decreasing as distance from the origin increases. Obviously such an analysis is only experimental, but in practice the estimates given by differential approximation are incredibly accurate. In tests where the true singularity structure of a generating function is independently known, the estimates from differential approximation are rarely off by more than the last decimal place. 
The counting sequence of pop-stacked permutations grows superexponentially [1, implying that its generating function has a singularity at the origin. Accordingly, we use differential approximation to analyze the exponential generating function. It predicts a number of singularities on the positive real axis, located at the values below.

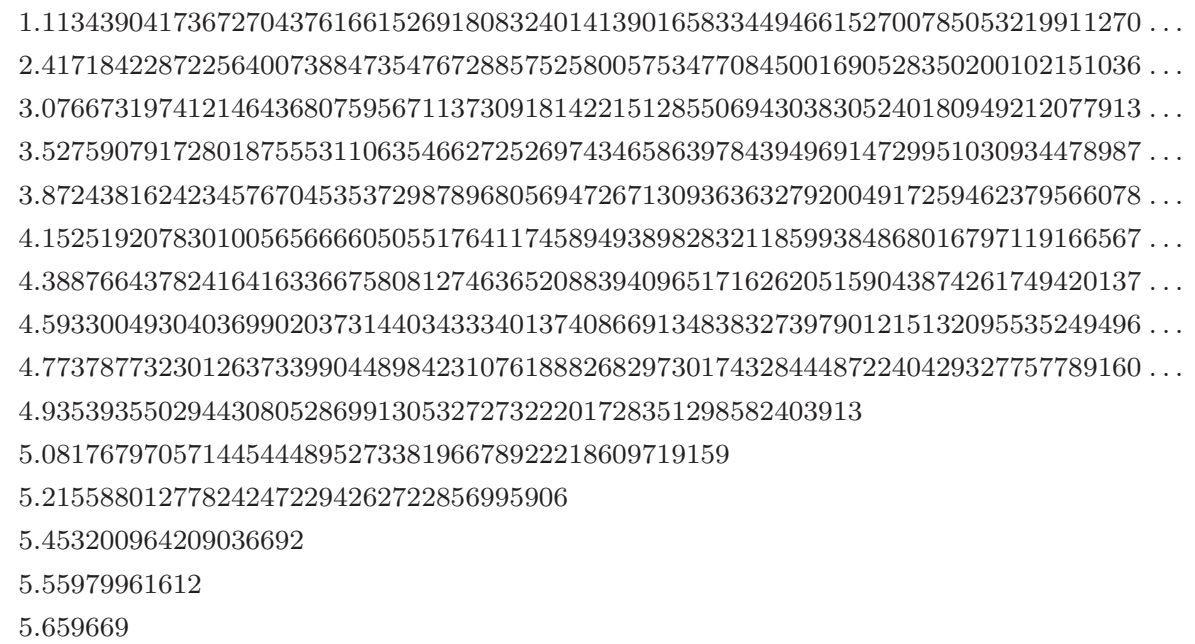

Each of these singularities is predicted to have critical exponent -1 , making them simple poles. The topmost 9 estimates have been truncated to fit on the page. In reality, they are given to many more decimal places - nearly 800 for the dominant singularity. More precise estimates could be obtained if desired. These results suggest that the exponential generating function may posses an infinite number of singularities. If true, this would imply the non-D-finiteness of both the ordinary and exponential generating functions.

Differential approximation also predicts several complex pairs of singularities, also simple poles, of which we'll list just a few.

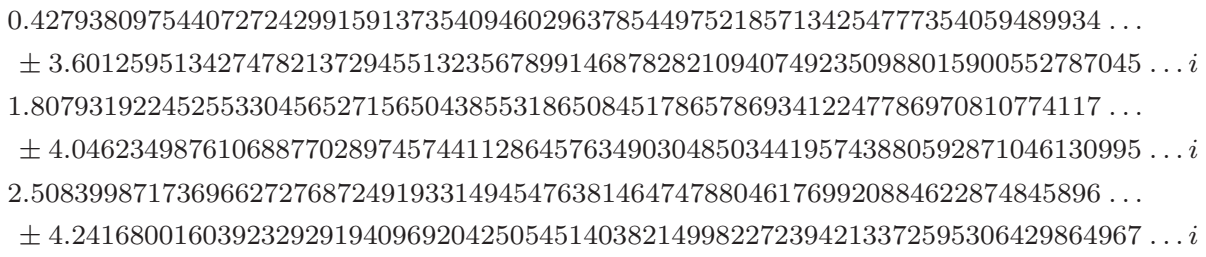

The dominant pole at $\mu \approx 1.11343904$ implies that the exponential growth rate of the counting sequence is

$$
\mu^{-1} \approx 0.8981183185746869695116759646856448 \ldots,
$$

implying that the asymptotic behavior of the number of pop-stacked permutations is

$$
a_{n} \sim C \cdot n ! \cdot(0.898118 \ldots)^{n} .
$$


Differential approximation does provide an estimate for the constant $C$ but this can be obtained numerically given the extremely accurate estimate for $\mu$. We find that

$$
C \approx 0.6956885490706357679957031687241101565741983507216179232324 \ldots
$$

giving the final asymptotic approximation

$$
a_{n} \sim(0.695688 \ldots) \cdot n ! \cdot(0.898118 \ldots)^{n} .
$$

Full decimal values for the approximated singularities and constants can also be found on GitHub [4].

Acknowledgements. Computations were performed on the Garpur cluster [8, a joint project between the University of Iceland and the University of Reykjavik funded by the Icelandic Centre for Research. We thank them for the use of their resources.

\section{References}

[1] Andrei Asinowski, Cyril Banderier, Sara Billey, Benjamin Hackl, and Svante Linusson. Pop-stack sorting and its image: Permutations with overlapping runs. To appear in Eurocomb 2019.

[2] David Avis and Monroe Newborn. On pop-stacks in series. Utilitas Math, 19(129-140):410, 1981.

[3] Anders Claesson and Bjarki Ágúst Guðmundsson. Enumerating permutations sortable by $k$ passes through a pop-stack. Adv. in Appl. Math., 108:79-96, 2019.

[4] Anders Claesson, Bjarki Ágúst Guðmundsson, and Jay Pantone. Enumerating the pop-stacked permutations. https://github.com/SuprDewd/pop-stacked-perms.

[5] Anthony J Guttmann. Asymptotic analysis of power-series expansions. Phase transitions and critical phenomena, 13:1-234, 1989.

[6] Anthony J Guttmann and Iwan Jensen. Series analysis. In Polygons, polyominoes and polycubes, volume 775 of Lecture Notes in Phys., pages 181-202. Springer, Dordrecht, 2009.

[7] Waldemar Hebisch and Martin Rubey. Extended rate, more GFUN. J. Symbolic Comput., 46(8):889-903, 2011.

[8] Garpur cluster. IHPC - Icelandic High Performance Computer - University of Iceland and Reykjavik University, 2019.

[9] Manuel Kauers. Guess: A Mathematica package for guessing multivariate recurrence equations. Research Institute for Symbolic Computation.

[10] Bruno Salvy and Paul Zimmermann. GFUN: A Maple package for the manipulation of generating and holonomic functions in one variable. $A C M$ Trans. Math. Softw., 20(2):163-177, June 1994. 
[11] Neil J. A. Sloane. The Online Encyclopedia of Integer Sequences. https://oeis.org, 2019. 